\title{
Recent Applications of \\ Nirenberg's Classical Ideas
}

\section{Communicated by Christina Sormani}

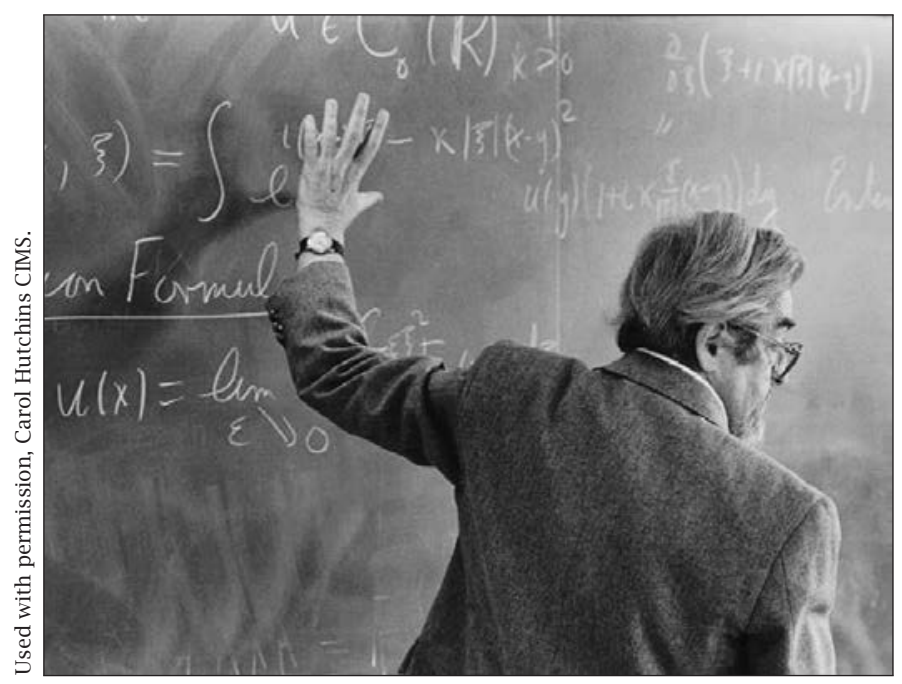

Louis Nirenberg teaching at the Courant Institute of Mathematical Sciences, New York University.

I N 2015, Louis Nirenberg and John F. Nash Jr. were awarded the Abel Prize "for striking and seminal contributions to the theory of nonlinear partial differential equations and its applications to geometric analysis." While many an article has been written describing Nirenberg's greatest works, here we have asked mathematicians to describe how they have applied Nirenberg's ideas in new and exciting ways.

We begin with Xavier Cabré, a former student of Nirenberg, who describes extensions of the Gidas-Ni-Nirenberg results on the symmetries of solutions to nonlinear elliptic partial differential equations. Alice Chang then describes recent work on Nirenberg's problem: prescribing the Gauss curvature on a sphere. Gregory Seregin discusses recent work on the Navier-Stokes problem applying the work of Caffarelli-Kohn-Nirenberg. Eric Carlen and Alessio

Christina Sormani is professor of mathematics at Lehman College and CUNY Graduate Center. Her email address is sormanic@ gmai1. com.

Xavier Cabré is ICREA Professor of Mathematics at the University of Politecnica de Catalunya. His email address is xavi er . cabre@ upc. edu.

For permission to reprint this article, please contact:

reprint-permission@ams.org.

DOI: http://dx.doi.org/10.1090/noti1332
Figalli describe the stability of the GNS inequality and recent applications of this result. Mu-Tao Wang and ShingTung Yau describe applications of Nirenberg's solution of the Weyl problem to general relativity.

Of course there are many, many more important applications of Nirenberg's work that we cannot touch on in this article. In fact, Nirenberg has been cited over 10,000 times by over 5,000 authors. We hope just to give a flavor of the new directions being taken with his work.

\section{Xavier Cabré}

\section{The Gidas-Ni-Nirenberg Theorem}

In 1979, Gidas, Ni, and Nirenberg [4] established an important result on monotonicity and symmetry of solutions to nonlinear second order elliptic PDEs (see Figure 1):

Theorem 1. Let $\Omega$ be a bounded smooth domain of $\mathbb{R}^{n}=$ $\mathbb{R} \times \mathbb{R}^{n-1}$ which is symmetric with respect to $\left\{\left(x_{1}, x^{\prime}\right): x_{1}=\right.$ $0\}$ :

if $\left(p_{1}, x^{\prime}\right) \in \Omega$, then the reflected point $\left(-p_{1}, x^{\prime}\right) \in \Omega$

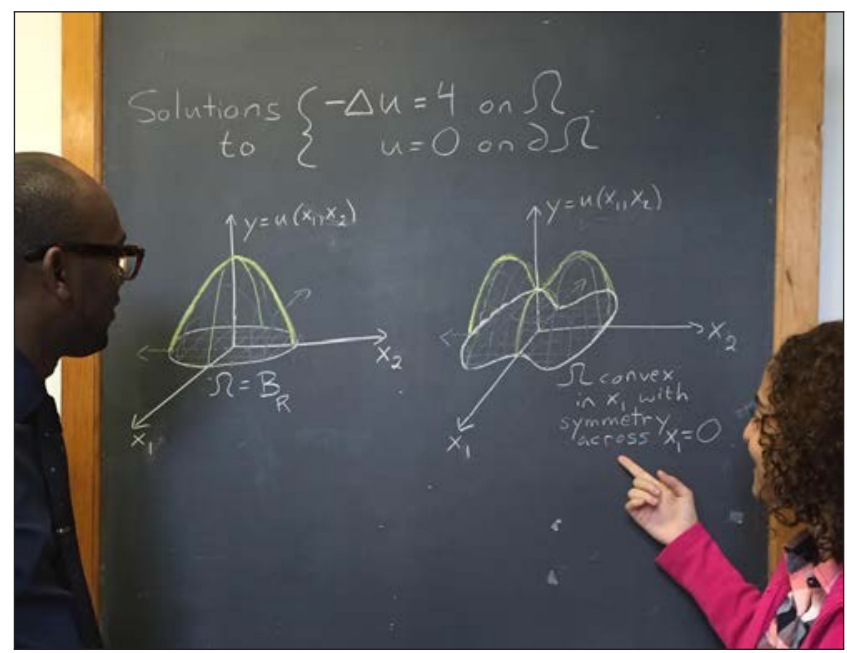

Figure 1. Students at the City University of New York discussing the symmetries of solutions as described in the Gidas-Ni-Nirenberg Theorem. 
and is convex in the $x_{1}$-direction:

$$
\begin{aligned}
& \text { if } p=\left(p_{1}, x^{\prime}\right) \in \Omega \text { and } q=\left(q_{1}, x^{\prime}\right) \in \Omega, \\
& \text { then the line segment } \overline{p q} \subset \Omega .
\end{aligned}
$$

Let $f$ be a locally Lipschitz function and $u$ be a bounded positive solution of

$$
-\Delta u=f(u) \text { in } \Omega \text { with } u=0 \text { on } \partial \Omega .
$$

Then $u$ is symmetric with respect to $\left\{x_{1}=0\right\}$ :

$$
u\left(p_{1}, x^{\prime}\right)=u\left(-p_{1}, x^{\prime}\right) \quad \forall\left(p_{1}, x^{\prime}\right) \in \Omega,
$$

and $u$ is monotone for $x_{1}>0$ :

$$
\partial_{x_{1}} u<0 \text { in } \Omega \cap\left\{x_{1}>0\right\} .
$$

In particular, if $\Omega=B_{R}$ is a ball, then $u$ is radially symmetric and

$$
\partial_{r} u<0 \quad \forall x \in B_{R} \backslash\{0\} \quad \text { where } r=|x| .
$$

The proof uses the maximum principle and is very flexible. It allows for extensions to some unbounded domains (in particular, all of $\mathbb{R}^{n}$ ), to fully nonlinear elliptic equations, to some nonlinearities $f(x, u)$ depending also on the variable $x \in \Omega$, as well as to some elliptic systems of equations. As a consequence, this is Louis Nirenberg's most cited paper. As he once said, "I made a living off the maximum principle."

If for a certain nonlinearity function, $f$, one knows that there is uniqueness of positive solution to the above boundary value problem (this happens for instance when $f$ is nonincreasing, since then the energy functional is convex), then the solution $u$ must be symmetric as an immediate consequence of the uniqueness. However, in many interesting applications (certain ground states in mathematical physics, curvature equations in conformal geometry), uniqueness does not hold, and the symmetry result requires a proof. Note also that positivity of the solution is needed in the assumptions. Indeed, the second Dirichlet eigenfunction of the Laplacian in a ball (like $\sin (x)$ on $[-\pi, \pi]$ ) satisfies $-\Delta u=\lambda_{2} u$, but it is not radially symmetric. In fact, it is odd with respect to one hyperplane through the center of the ball.

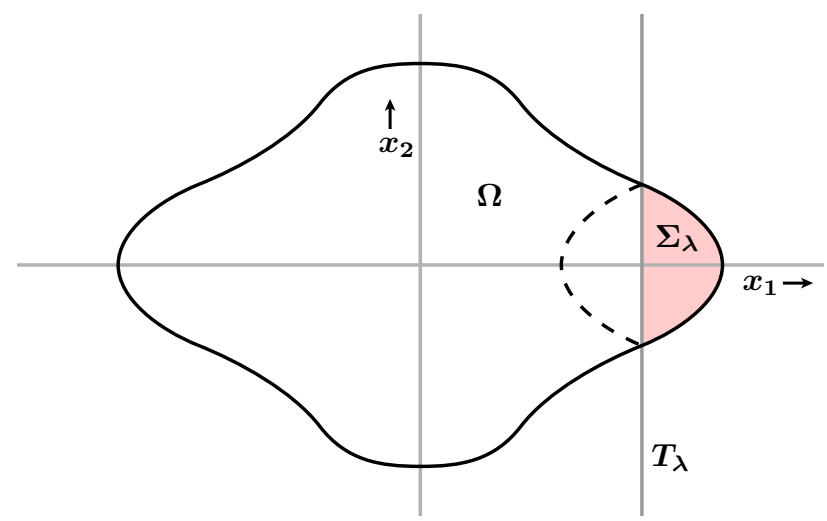

Figure 2. In the Alexandrov method, one reflects domains $\Sigma_{\lambda}$ across vertical hyperplanes $T_{\lambda}$ that move continuously from the right to the middle.
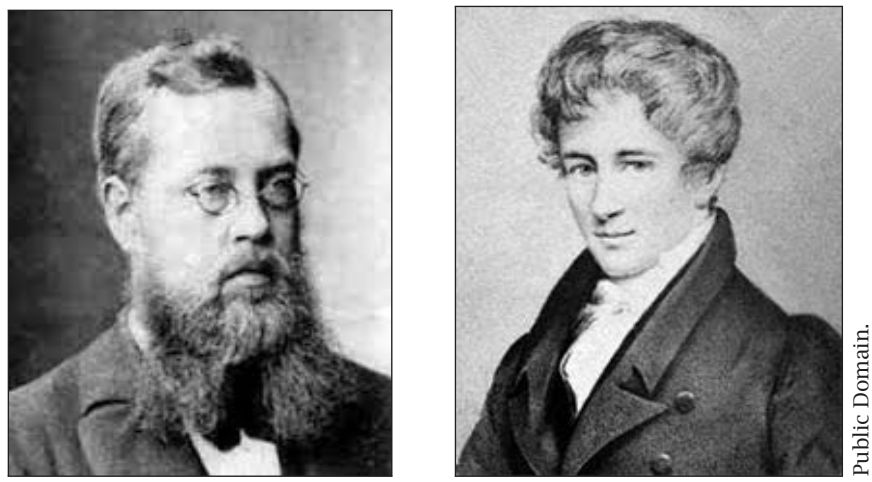

Sophus Lie and Niels Henrik Abel, the two Norwegian mathematicians who inspired the Abel Prize.

The proof of the Gidas-Ni-Nirenberg result uses the powerful Alexandrov moving planes method. Alexandrov developed this method circa 1955 in a series of papers to establish (among other results) that spheres are the only embedded, bounded, and connected hypersurfaces of $\mathbb{R}^{n}$ with constant mean curvature. In 1971, J. Serrin [6] used the method to prove that balls are the only bounded smooth domains that admit a solution to certain overdetermined boundary value problems.

In the Alexandrov method, one considers the planes $T_{\lambda}=\left\{x_{1}=\lambda\right\}$, the domains $\Sigma_{\lambda}=\Omega \cap\left\{x_{1}>\lambda\right\}$, and the reflected function $u^{\lambda}(x)=u\left(2 \lambda-x_{1}, x^{\prime}\right)$ (the reflection of $u$ across $T_{\lambda}$ ) as in Figure 2. One shows that $u<u^{\lambda}$ in $\Sigma_{\lambda}$ for all $0<\lambda<\lambda^{*}:=\sup _{\Omega} x_{1}$. One starts proving this for $\lambda$ close to $\lambda^{*}$ and then showing, through a continuity argument, that the same holds all the way down to $\lambda=0$. The two ingredients used here are the Hopf boundary lemma and the strong maximum principle. They are applied to the difference $u^{\lambda}-u$, which satisfies a linear equation, since $u^{\lambda}$ solves the same nonlinear equation as the one for $u$.

\section{Sidebar 1. Abel Prize Winners}

2015: John Forbes Nash, Jr. and Louis Nirenberg

2014: Yakov Sinai

2013: Pierre Deligne

2012: Endre Szemerédi

2011: John Milnor

2010: John Tate

2009: Mikhail Leonidovich Gromov

2008: John G. Thompson and Jacques Tits

2007: S. R. Srinivasa Varadhan

2006: Lennart Carleson

2005: Peter Lax

2004: Michael Atiyah and Isadore Singer

2003: Jean-Pierre Serre 
The use of the Hopf boundary lemma required some regularity of the domain $\Omega$ in the original paper of GidasNi-Nirenberg, and thus symmetry in domains such as a square in the plane was left open. A new approach was then developed by Berestycki and Nirenberg in 1991. It replaced the use of the Hopf lemma by a maximum principle in "small domains", domains such as $\Sigma_{\lambda}$ when $\lambda$ is very close to $\lambda^{*}$ or $\Sigma_{\lambda} \backslash K$, where $\lambda$ is arbitrary and $K$ is a sufficiently large compact set inside $\Sigma_{\lambda}$. In this way, they succeeded in proving symmetry in nonsmooth domains such as cubes. In addition, Berestycki and Nirenberg [1] introduced the sliding method: a new and powerful tool leading to the monotonicity of solutions in certain problems (and sometimes to their uniqueness or to their oddness). It consists of comparing the solution $u$ with the slided (or translated) solutions $u_{\lambda}(x):=u\left(x_{1}+\lambda, x^{\prime}\right)$.

Within elliptic PDEs there has been great activity in these last years on equations involving fractional Laplacians or related nonlocal operators, for instance, the generators of Lévy stable diffusion processes. They arise when studying anomalous diffusions in plasmas, chemical reactions in liquids, geophysical fluid dynamics, population dynamics, and finance. The Gidas-Ni-Nirenberg result has recently been extended to many of these fractional operators. For example, Birkner, López-Mimbela, and Wakolbinger [2] extended it to the Dirichlet problem in a ball for the fractional Laplacian, which reads $(-\Delta)^{s} u=f(u)$ in $B_{R}$ and $u=0$ in $\mathbb{R}^{n} \backslash B_{R}$ (the complement of $B_{R}$ ).

In 2010, Jinggang Tan and I [3] used the moving planes method to show symmetry and monotonicity for the spectral square root of the Laplacian on a bounded domain with zero Dirichlet boundary conditions. That is, we considered positive solutions to

$$
A_{1 / 2} u=f(u) \text { in } \Omega \text { with } u=0 \text { on } \partial \Omega .
$$

To define $A_{1 / 2}$, we took the eigenvalues, $\lambda_{k}$, and eigenfunctions, $\varphi_{k}$, of the Laplacian in $\Omega$ :

$$
-\Delta \varphi_{k}=\lambda_{k} \varphi_{k} \text { in } \Omega \text { with } \varphi_{k}=0 \text { on } \partial \Omega \text {. }
$$

Then, given the expansion $u=\sum_{k=1}^{\infty} c_{k} \varphi_{k}$, we set $A_{1 / 2} u=$ $\sum_{k=1}^{\infty} c_{k} \lambda_{k}^{1 / 2} \varphi_{k}$.

This is a nonlocal problem, but we transformed it into a local problem in one more dimension by viewing it as a Dirichlet-to-Neumann operator: given $u$ in $\Omega$, find its harmonic extension $w$ in $\Omega \times[0, \infty)$ with zero Dirichlet condition on $\partial \Omega \times[0, \infty)$. Then we have $A_{1 / 2} u=\left.\frac{\partial w}{\partial \nu}\right|_{\Omega \times\{0\}}$. We were then able to apply the moving planes method, combined with a maximum principle in domains of small measure, to obtain the Gidas-Ni-Nirenberg type symmetry results for locally Lipschitz nonlinearities $f$. In particular, if $\Omega$ is a ball, we proved that any positive solution $u$ to (2) is rotationally symmetric and $u_{r}<0$ within the ball.

\section{References}

[1] H. BERESTYCKI and L. NiRENBERG, On the method of moving planes and the sliding method, Bol. Soc. Brasil. Mat. (N.S.) 22 (1991), no. 1, 1-37.

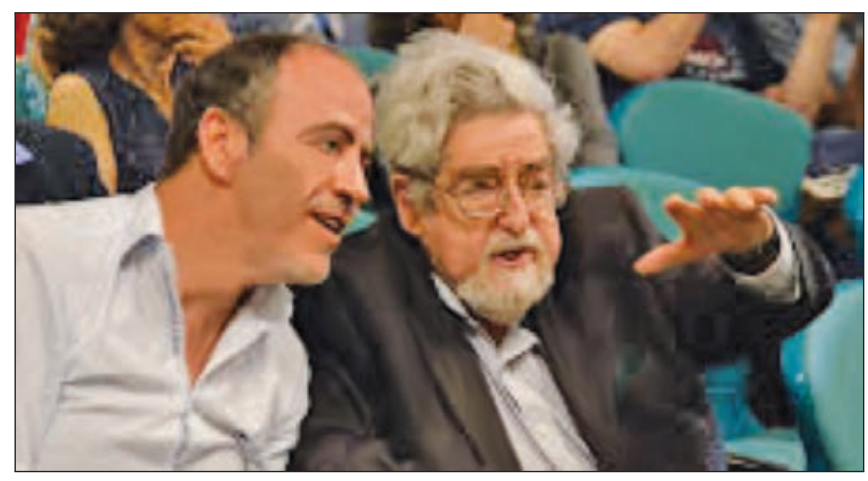

Xavier Cabré with Louis Nirenberg in 2015.

[2] M. Birkner, J. A. López-MimbelA, and A. WAKOlbinger, Comparison results and steady states for the Fujita equation with fractional Laplacian, Ann. Inst. H. Poincaré Anal. Non Linéaire 22 (2005), 83-97.

[3] X. CABRÉ and JingGang TAN, Positive solutions of nonlinear problems involving the square root of the Laplacian, $A d v$. Math. 224 (2010), no. 5, 2052-2093.

[4] B. Gidas, Wei Ming Ni, and L. Nirenberg, Symmetry and related properties via the maximum principle, Comm. Math. Phys. 68 (1979), no. 3, 209-243.

[5] J. SERRIN, A symmetry problem in potential theory, Arch. Rational Mech. Anal. 43 (1971), 304-318.

\section{Sun-Yung Alice Chang}

\section{Nirenberg's Problem}

In 1970, Louis Nirenberg proposed the problem of "prescribing Gaussian curvature on the sphere." This question has led to one of the most active and exciting subfields of geometric analysis. The penetrating question raised by Nirenberg is, What are the functions, $K$, which can arise as the Gaussian curvature function, $K_{g}$, for some Riemannian metric, $g$, on the sphere?

On the standard two-dimensional sphere, by the GaussBonnet formula, one has

$$
\int_{S^{2}} K_{g} d v_{g}=4 \pi
$$

Thus any such Gaussian curvature function $K_{g}$ must be positive somewhere on the sphere. See Figure 4 for a surface with positive Gaussian curvature everywhere and this issue's article, “WHAT IS...Gauss Curvature?" (page 144), for more information about Gaussian curvature.

At the time that Nirenberg's problem was originally posed, it was considered that this might be the only restriction on the function $K=K_{g}$. Then in 1974, KazdanWarner ([4]) indicated that this is not the case, that $K=K_{g}$ must satisfy another condition:

$$
\int_{S^{2}}\left\langle\nabla K, \nabla x_{i}\right\rangle d \mu_{g}=0
$$

Sun-Yung Alice Chang is Eugene Higgins Professor of Mathematics at Princeton University. Her email address is chang@ math.princeton. edu. 




Louis Nirenberg, Alice Chang and Paul Yang in 2015.

where $x_{i}, i=1,2,3$, are the coordinate functions of the sphere. In particular, the Nirenberg problem has no solution for $K(p)=f\left(x_{1}(p)\right)$ where $f$ is monotone.

Nirenberg's problem was first studied in the 1971 doctoral thesis of D. Koutroufiotis, a PhD student of Nirenberg. Here $g$ was assumed to be conformal to the standard metric, $g_{0}$, on the two-dimensional sphere, $\mathbb{S}^{2}$. If one writes $g=e^{2 \omega} g_{0}$, then the problem is equivalent to solving

$$
-\Delta \omega+1=K e^{2 \omega} \text { on } \mathbb{S}^{2},
$$

where $\Delta$ is the Laplacian with respect to the standard metric $g_{0}$ on $\mathbb{S}^{2}$. Koutroufiotis proved that when $K$ is antipodally symmetric and sufficiently close to 1 , then there is a solution, $g$, to the problem $K=K_{g}$.

In 1973 Moser proved that for any antipodally symmetric function $K$ which is positive somewhere, there is a solution to this original version of the Nirenberg problem. This important breakthrough was founded upon Moser's inequality, a sharp version of a limiting Sobolov imbedding result by Trudinger. To prove his result, Moser first observed that solutions $\omega$ to (5) are the critical points of the functional

$$
F_{K}[\omega]=\log \frac{1}{4 \pi} \int_{\mathbb{S}^{2}} K e^{2 \omega} d \mu_{0}-\frac{1}{4 \pi} \int_{\mathbb{S}^{2}}\left(|\nabla \omega|^{2}+2 \omega\right) d \mu_{0} .
$$

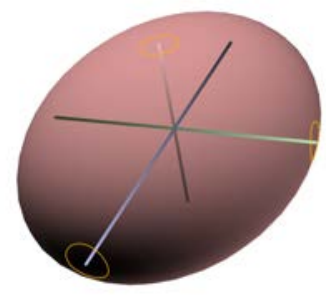

Figure 3. An ellipsoid shaded by Gaussian curvature.
Moser's inequality provides a bound on the terms in the right-hand side of this inequality. One may then find a solution to the Nirenberg problem as a limit of a sequence of functions via a minimax procedure using this bound to prove the sequence converges. This method has led to many additional partial solutions of the Nirenberg problem, including results by the author with P. Yang, K. C. Chang with J. Q. Liu, Z. C. Han, and others.

In particular, the author and Paul Yang proved the following theorem in 1987: Let $K$ be a positive smooth function with only nondegenerate critical points. Suppose there are at least two local maxima of $K$ and suppose that at all saddle points, $p$, of $K$, we have $\Delta_{g_{0}} K(p)>0$. Then $K$ is the Gauss curvature $K_{g}$ of a metric $g$ conformal to $g_{0}$. In joint work with Gursky and Yang appearing in 1993, the author provided an "index formula" for $K$ to be the Gaussian curvature of a metric $g$ conformal to $g_{0}$. In 2005, Michael Struwe re-proved our 1987 theorem using flow methods and produced an example demonstrating that the hypotheses are in some sense the best possible. More precisely, Struwe constructed functions, $K$, having exactly two local maxima and one saddle point, $p$, where $\Delta K(p)<$ 0 , which cannot be realized as curvature functions of conformal metrics on the two-dimensional sphere.

In another direction, the problem of prescribing Gaussian curvature further developed into the problem of “prescribing $Q$-curvature". The connection of $Q$-curvature to the Gauss curvature was first pointed out by T. Branson in 1995. For example, on 4-spheres, through the GaussBonnet formula, one has $\int_{S^{4}} Q_{g} d v_{g}=32 \pi^{2}$. It turns out that $Q$-curvatures on compact manifolds of even dimensions share many of the same analytic properties as the Gaussian curvature on compact surfaces, and the tool introduced by Moser can be effectively applied. Study of $Q$-curvature has become an important topic in conformal geometry, with applications to problems in Ads/CFT theory (by Graham-Zworski and Fefferman-Graham) and geometry (by Chang-Gursky-Yang and others). The field is active and developing (with important papers in 2013 by Branson-Fontana-Morpurgo and by Case-Yang).

\section{On a Personal Note:}

My relationship with Louis began as that of a mentor and later a respected, well-admired friend. All the time I have spent with him-either discussing mathematics or just chatting-has been wonderful. It is like the saying in Chinese, "You are enjoying the breeze of the spring," a wonderful experience in life.

\section{References}

[1] T. BRANSON, Sharp inequality, the functional determinant and the complementary series, Trans. Amer. Math. Soc. 347 (1995), 3671-3742.

[2] S. Y. A. ChAng and P. C. YANG, Prescribing Gaussian curvature on $S^{2}$, Acta Math. 159 (1987), 215-259.

[3] C. FeFFERman and C. R. GRAHAM, $Q$-curvature and Poincaré metrics, Math. Res. Lett. 9 (2002), nos. 2 and 3, 139-152.

[4] J. KAZDAN and F. WARNER, Existence and conformal deformation of metrics with prescribed Gaussian and scalar curvature, Ann. of Math. 101 (1975) 317-331. 


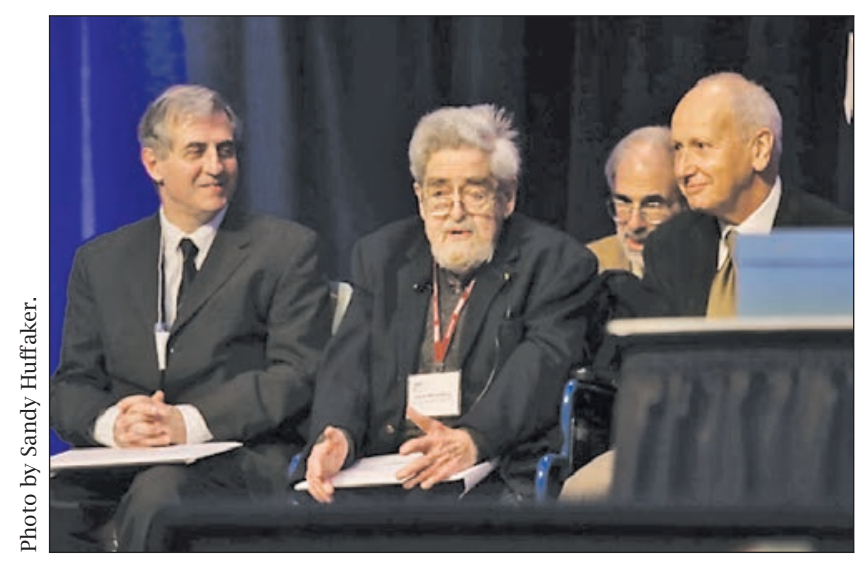

Louis Nirenberg (center) and Luis Caffarelli (right) at the Joint Prize Ceremony of JMM 2014, Baltimore.

[5] J. Moser, On a nonlinear problem in differential geometry, Dynamical Systems Symposium (M. Peixoto, ed.) Academic Press, NY, 1973, pp. 273-280.

[6] M. STRuwe, A flow approach to Nirenberg's problem, Duke Math. J. 128 (2005), no. 1, 19-64.

\section{Gregory Seregin}

\section{Caffarelli-Kohn-Nirenberg and the Navier-Stokes Problem}

One of the main problems in the field of mathematical hydrodynamics is a global well-posedness of the initial boundary value problem for the Navier-Stokes equations: (6) $\partial_{t} u+u \cdot \nabla u-\Delta u+\nabla p=f$ and $\operatorname{div} u=0$ on $\Omega \times[0, \infty)$, with the value of $u$ prescribed on $\Omega \times\{0\}$ and on $\partial \Omega \times[0, \infty)$. Here $u$ is the velocity field of the fluid and $p$ is the pressure. See Figure 4.

\section{The conceptual novelty of this paper is their explicit introduction of a new notion of suitable weak solutions to the Navier-Stokes equations}

One of the possible ways to attack this question is to consider the so-called weak Leray-Hopf solutions. Such solutions have finite energy and exist globally, and, as observed by J. Leray in 1934, their uniqueness would follow from their smoothness. In the 1970s Scheffer proved the existence of such solutions, $u$, for all time, such that the vorticity, $\nabla \times u$, is continuous outside a set of finite one-dimensional

Hausdorff measure in space.

Gregory Seregin is professor of mathematics at St. Hilda's College, University of Oxford. His email address is seregin@maths. ox.ac.uk.
The 1982 Caffarelli-Kohn-Nirenberg paper is one of the most important steps toward understanding whether or not the Navier-Stokes equations provide a deterministic description of the flow of a viscous incompressible fluid. The conceptual novelty of this paper is their explicit introduction of a new notion of suitable weak solutions to the Navier-Stokes equations in space-time domains. Louis Nirenberg and his co-authors focus on differentiability properties of these suitable weak solutions rather than consider a global setting with initial and boundary conditions. This makes the problem of regularity in a sense a local one.

Observe first that the Navier-Stokes equations are invariant under parabolic rescalings. That is, if $u(x, t)$ and $p(x, t)$ solve (6) for $f(x, t)$, then $u_{\lambda}(x, t)=\lambda u\left(\lambda x, \lambda^{2} t\right)$ and $p_{\lambda}(x, t)=\lambda^{2} p\left(\lambda x, \lambda^{2} t\right)$ also satisfy (6) for $f_{\lambda}(x, t)=$ $\lambda^{3} f\left(\lambda x, \lambda^{2} t\right)$. Thus it is natural to consider parabolic cylinders:

$$
Q_{r}(x, t)=\left\{(y, \tau):|y-x|<r, t-r^{2}<\tau<t\right\} .
$$

Then one may define the one-dimensional Hausdorff measure using these parabolic cylinders, $P^{1}$.

Caffarelli-Kohn-Nirenberg prove that their suitable weak solutions are smooth away from a singular set $S$ with $P^{1}(S)=0$. They consider a pair $u(x, t)$ and $p(x, t)$ to be a suitable weak solution on $Q_{r}(x, t)$ if

$$
\sup _{\tau \in\left(t-r^{2}, t\right)} \int_{B_{r}(x)}|u(y, \tau)|^{2} d y+\int_{Q_{r}(x, t)}|\nabla u|^{2} d y d \tau<\infty,
$$

if $p \in L_{\frac{5}{4}}\left(Q_{r}(x, t)\right)$, and if $u$ and $p$ satisfy the classical Navier-Stokes system in the sense of distributions on $Q_{r}(x, t)$ :

$$
\begin{aligned}
& 2 \int_{Q_{r}(x, t)}|\nabla u|^{2} \varphi d y d \tau \\
& \quad \leq \int_{Q_{r}(x, t)}\left[|u|^{2}\left(\partial_{\tau} \varphi+\Delta \varphi\right)+u \cdot \nabla \varphi\left(|u|^{2}+2 p\right)\right] d y d \tau
\end{aligned}
$$

for any nonnegative smooth function $\varphi$ compactly supported in $Q_{r}(x, t)$. The space for pressure can be taken slightly differently in order to simplify statements, and

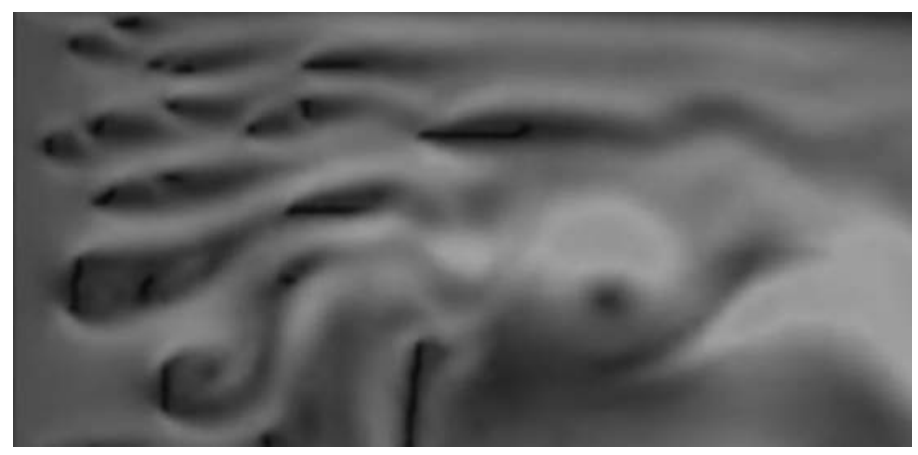

Figure 4. A numerical implementation of Navier-Stokes by Florian de Vuyst. Complete videos of the flow can be found linked from his webpage devuyst.perso.math.cnrs.fr. 
such a space is $L_{\frac{3}{2}}\left(Q_{r}(x, t)\right)$. With this minor simplification and the assumption $f=0$, one important theorem in Caffarelli-Kohn-Nirenberg may be restated as follows:

Theorem 2. If $u$ and $p$ are a suitable weak solution to the Navier-Stokes equations in $Q_{r}(x, t)$, then there exist positive universal constants $\varepsilon$ and $c_{k}, k=0,1, \ldots$, such that if

$$
\frac{1}{r^{2}} \int_{Q_{r}(x, t)}\left(|u|^{3}+|p|^{\frac{3}{2}}\right) d y d \tau<\varepsilon,
$$

then $\left|\nabla^{k} u(y, \tau)\right| \leq c_{k} / r^{k+1}$ for any $(y, \tau) \in \bar{Q}_{r / 2}(x, t)$ and any $k \in \mathbb{N}$.

In fact, the linear theory implies that all functions $\nabla^{k} u$ are Hölder continuous in the closure of $Q_{r / 2}(x, t)$. The exponent of Hölder continuity is related to the choice of a functional space for the pressure.

Let me give a simple application of Theorem 2 that arose when proving the Ladyzhenskaya-Prodi-Serrin condition of regularity by rescaling around a possible singular point. After a certain rescaling, there are sufficiently smooth functions $u$ and $p$ that satisfy the Navier-Stokes equations in $\left.\mathbb{R}^{3} \times\right]-\infty, 0\left[\right.$. Suppose that $\|u\|_{L_{5}\left(\mathbb{R}^{3} \times\right]-\infty, 0[)}$ is bounded. Let us prove a Liouville-type theorem saying that $u=0$. Given $\mu>0$, we can find $A<0$ such that

$$
\int_{-\infty}^{A} \int_{\mathbb{R}^{3}}\left(|u|^{5}+|p|^{\frac{5}{2}}\right) d x d t<\mu .
$$

Fix any $z_{0}=\left(x_{0}, t_{0}\right)$ with $t_{0} \leq A$. Then for any $R>0$,

$$
\begin{aligned}
& \frac{1}{R^{2}} \int_{Q_{R}\left(z_{0}\right)}\left(|u|^{3}+|p|^{\frac{3}{2}}\right) d x d t \\
& \quad \leq c\left(\int_{Q_{R}\left(z_{0}\right)}\left(|u|^{5}+|p|^{\frac{5}{2}}\right) d x d t\right)^{\frac{3}{5}} \leq c \mu^{\frac{2}{5}} .
\end{aligned}
$$

If $\mu^{\frac{2}{5}} \leq \varepsilon$, then one can use Theorem 2 and conclude that $\left|u\left(z_{0}\right)\right| \leq c_{0} / R$. Taking $R \rightarrow \infty$, we can deduce that $u=0$ in $\left.\mathbb{R}^{3} \times\right]-\infty, A[$. We then split the interval $] A, 0[$ into sufficiently small pieces and sequentially exclude the corresponding layers in order to complete the proof that $u=0$.

A more sophisticated application of this theorem appears in my 2012 paper on the potential blowup for Navier-Stokes equations and in an earlier paper of mine with Escauriaza and Šverák appearing in 2003. In the more recent paper, which improves upon the work in the earlier paper, one considers weak Leray-Hopf solutions to Navier-Stokes equations on $\mathbb{R}^{3} \times[0, T)$ with initial data $u(x, 0)=u_{0}(x)$ smooth with compact support. If the finite energy solution arising from $u_{0}$ blows up at time $T<\infty$, then

$$
\lim _{t \rightarrow T^{-}}\|u(x . t)\|_{L^{3}\left(\mathbb{R}^{3}\right)}=\infty .
$$

In order to prove this result, one needs to apply a backward uniqueness theorem for a certain parabolic differential inequality. We need to provide a decay at the spatial infinity for some ancient (backward) solution to the Navier-Stokes equations. Recall that an ancient solution is one which is well defined for all time $t \in(-\infty, 0)$ and is found by taking a parabolic rescaling around the developing singularity. In particular, having in our hands a finite global $L_{3}$-norm, we can make the tails of the corresponding integrals small enough and then apply the Caffarelli-Kohn-Nirenberg theorem stated above.

In a sense, the results of the Caffarelli-Kohn-Nirenberg paper seem to be optimal for a local solution. To go further, one needs additional information on the global level, i.e., on the level of initial boundary value problems for the Navier-Stokes system. In his interview with the Notices of AMS in 2002, Nirenberg pointed out that further applications of harmonic analysis could be useful toward making additional advances.

\section{References}

[1] L. CAFfarelli, R. KoHn, and L. NirenberG, Partial regularity of suitable weak solutions of the Navier-Stokes equations, Comm. Pure Appl. Math. 35 (1982), no. 6, 771-831.

[2] L. EscauriazA, G. SEREgIN, and V. ŠVERÁK, $L_{3, \infty}$-solutions of Navier-Stokes equations and backward uniqueness (Russian), Uspekhi Mat. Nauk. 58 (2003), no. 2(350), 3-44; translation in Russian Math. Surveys 58 (2003), no. 2, 211-250.

[3] J. LERAY, Sur le mouvement d'un liquide visqueux emplissant l'espace, Acta Math. 63 (1934), 193-248.

[4] G. SEREGIN, A certain necessary condition of potential blow up for Navier-Stokes equations, Comm. Math. Phys. 312 (2012), no. 3, 833-845.

\section{Eric Carlen and Alessio Figalli}

\section{Stability of the Gagliardo-Nirenberg Sobolev Inequality}

A central focus of Nirenberg's research has been partial differential equations. An important tool in their study consists of using functional inequalities that control some $L^{q}$ norm of a function in terms of the norm of its derivatives.

The Sobolev inequalities are the classic example. Let

$$
\|u\|_{q}=\left(\int_{\mathbb{R}^{n}}|u(x)|^{q} \mathrm{~d} x\right)^{1 / q},
$$

for $1 \leq q<\infty$, denote the $L^{q}$ norm of a function $u$. Then, for $1 \leq p<n$, there exists a constant $C(n, p)$ such that for all smooth functions $u: \mathbb{R}^{n} \rightarrow \mathbb{R}$ vanishing at infinity,

$$
\|u\|_{p^{*}} \leq C(n, p)\|\nabla u\|_{p}, \quad p^{*}:=\frac{n p}{n-p} .
$$

The value of $p^{*}$ is determined by scaling: inserting $u(\lambda x)$, $\lambda>0$, into the inequality above, the $L^{p^{*}}$ norm is the only one for which both sides are proportional to the same power of $\lambda$.

As useful as these inequalities are, in the late 1950s mathematicians studying elliptic and parabolic regularity understood the need for an extension of the Sobolev inequalities. The first example in this direction appeared

Eric Carlen is professor of mathematics at Rutgers University. His email address is carlen@math. rutgers . edu.

Alessio Figalli is professor of mathematics at the University of Texas at Austin. His email address is figa11i@math. utexas.edu. 


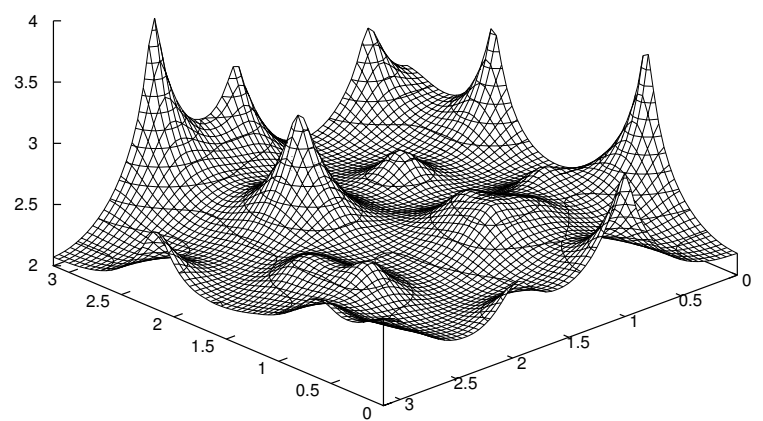

Figure 5. The Keller-Segel model of chemotaxis shows the characteristic clumping. Numerical implementation by Ibrahim Fatkullin.

in Nash's famous paper about the regularity of solutions to parabolic equations with measurable coefficients [5], where he showed what is now called the "Nash inequalities": ${ }^{1}$

$$
\|u\|_{2}^{2} \leq C_{n}\|\nabla u\|_{2}^{n /(n+2)}\|u\|_{1}^{2 /(n+2)} .
$$

Basically at the same time as Nash, both Gagliardo and Nirenberg independently proved a general family of inequalities including both the Sobolev inequalities and the Nash inequality as special cases. In their form, these can be stated as follows.

Theorem 3 (Gagliardo-Nirenberg). Let $u: \mathbb{R}^{n} \rightarrow \mathbb{R}$ be a smooth function vanishing at infinity. Then, for any $1 \leq$ $p \leq n$ and $1 \leq r \leq q \leq p^{*}$,

$$
\|u\|_{r} \leq C(n, p, r, s)\|\nabla u\|_{p}^{\theta}\|u\|_{q}^{1-\theta},
$$

where $\theta \in[0,1]$ satisfies $1 / r=\theta(1 / p-1 / n)+(1-\theta) / q$.

Again, the relation beween the exponents and $\theta$ is governed by scaling. Note that the Gagliardo-Nirenberg Sobolev inequalities hold and were proved by them for higher derivatives of $u$ and a larger set of exponents as well. Here we stated only the most frequently used cases.

The initial applications of these inequalities to regularity problems did not require knowledge of the sharp constant $C(n, p, r, s)$. However, the Gagliardo-Nirenberg Sobolev inequalities have since found many other applications for which this knowledge is useful and has motivated work to determine the sharp constants and optimizers.

While it is relatively easy to show that minimizers exist and are radially symmetric, finding the sharp constants and the explicit form of the minimizers has only been done in a few cases. Carlen and Loss [2] did this for the Nash inequalities, and Del Pino and Dolbeault [3] did this for the one-parameter family of inequalities corresponding to the case $p=2, q=t+1$, and $r=2 t$. The work in [2] was motivated by a question raised by Kato concerning the 2-dimensional Navier-Stokes equation, while the authors of [3] used the sharp form of these GN inequalities to study nonlinear diffusions.

${ }^{1}$ In his paper, Nash thanks Elias Stein for the proof (using the Fourier transform) of this inequality.
Recent works have shown that going beyond the characterization of the optimizers and proving a stability result can actually give further information on the rate of approach to the equilibrium for such nonlinear diffusion equations. For example, consider the Gagliardo-Nirenberg Sobolev inequality in the particular case $n=2, p=2, r=4$, $s=6$. In this case, the sharp form found by Del Pino and Dolbeault takes the form

$$
\pi\|u\|_{6}^{6} \leq\|\nabla u\|_{2}^{2}\|u\|_{4}^{4},
$$

and there is equality if and only if, up to a dilation, $u(x)=v_{\lambda, x_{0}}(x):=\lambda^{1 / 3}\left(1+\lambda^{2}\left|x-x_{0}\right|^{2}\right)^{-1 / 2}$.

We now define the quantity

$$
\delta_{G N}[u]:=\|\nabla u\|_{2}^{2}\|u\|_{4}^{4}-\pi\|u\|_{6}^{6} .
$$

Note that by the above, $\delta_{G N}[u]$ is nonnegative. Furthermore, if $\delta_{G N}[u]=0$, then $u$ is an optimizer. We say this last fact is "stable" if $\delta_{G N}[u]<\varepsilon$ for $\varepsilon>0$ sufficiently small implies that $u$ is close to an optimizer in some suitable norm.

In 2013 we proved the following quantitative stability estimate:

Theorem 4. Let $u: \mathbb{R}^{n} \rightarrow \mathbb{R}$ be a smooth nonnegative function vanishing at infinity such that $\|u\|_{6}=\left\|v_{1,0}\right\|_{6}$. Then there exist universal constants $K_{1}, \delta_{1}>0$ such that whenever $\delta_{G N S}[u] \leq \delta_{1}$,

$$
\inf _{\lambda>0, x_{0} \in \mathbb{R}^{2}}\left\|u^{6}-v_{\lambda, x_{0}}^{6}\right\|_{1} \leq K_{1} \sqrt{\delta_{G N}[u]} .
$$

In the same paper, we applied this result to obtain a quantitative bound to the rate of convergence of equilibrium for the critical mass Keller-Segel equation.

This equation was introduced by Evelyn Keller and Lee Segel in 1970 to model the aggregation exhibited by slime molds, a name for several types of eukaryotic cells that, depending on environmental circumstances and life cycle, either live freely as individual cells or else aggregate into tightly bound units. The mechanism driving aggregation in a population of free cells is chemotaxis: the cells emit a chemical attractant that draws the population together, while the diffusive motion of free cells promotes dispersion of the population (see Figure 5). The equation is mathematically and biologically interesting because there is a critical balance between the dissipative effects of diffusion and the accretive effects of chemotaxis, and a sharp Gagliardo-Nirenberg-Sobolev inequality together with related inequalities determines what happens at the threshold where these effects are balanced.

It is interesting that the papers of Gagliardo, Nirenberg, and Nash all appeared in 1958, and their utility for questions concerning rates of smoothing and decay of norms for solutions of parabolic equations, which has been the source of much of their continuing interest, was already realized at this time. A posteriori, these results had a tremendous impact in surprisingly many applications and have inspired-and still do inspire-an enormous number of authors. 


\section{References}

[1] ERIC A. CARLEN and Alessio FigAlli, Stability for a GNS inequality and the log-HLS inequality, with application to the critical mass Keller-Segel equation, Duke Math. J. 162 (2013), no. 3, 579-625.

[2] ERIC A. CARLEN and Michael Loss, Sharp constant in Nash's inequality, Int. Math. Res. Notices, Vol. 1993, 1993, 213-215.

[3] Manuel Del Pino and Jean Dolbeault, Best constants for Gagliardo-Nirenberg inequalities and applications to nonlinear diffusions, J. Math. Pures Appl. (9) 81 (2002), no. 9, 847-875.

[4] Emilio Gagliardo, Proprietà di alcune classi di funzioni in più variabili (Italian), Ricerche Mat. 7 (1958), 102-137.

[5] JoHn NASH, Continuity of solutions of parabolic and elliptic equations, Amer. J. Math. 80 (1958), 931-954.

[6] LOUIS NiRENBERG, On elliptic partial differential equations, Ann. Scuola Norm. Sup. Pisa (3) 13 (1959), 115-162.

\section{Mu-Tao Wang and Shing-Tung Yau}

\section{Isometric Embeddings of Surfaces}

In 1916, Weyl proposed the conjecture that every closed surface with positive Gauss curvature can be isometrically embedded into Euclidean 3-space. Moreover, this embedding should be unique up to an isometry of the Euclidean 3-space. Weyl's problem can be considered as an existence and uniqueness problem for a global elliptic equation, and he contributed an important estimate of the second fundamental form. This was a major global problem in classical geometry in the first half of the twentieth century. Lewy solved Weyl's problem for real analytic metrics in 1932.

It was not until 1954 that Nirenberg and Pogorelov solved Weyl's problem for smooth metrics independently and using very different methods. While some of their work depends on the works of Morrey in 1938 on the general theory of elliptic equations, these are spectacular papers. Nirenberg's paper also includes a solution to the Minkowski problem. Both results have inspired much of the later work on global elliptic problems.

The work of Nirenberg-Pogorelov also has important applications in general relativity. The question of defining and understanding the concept of quasi-local mass had been a central topic since the works of Hawking and Penrose. The idea of defining mass in general relativity went all the way back to Einstein. It was difficult because the theory of general relativity is a nonlinear theory where gravity is dictated by a tensor, while Newtonian gravity is basically linear and depends on a scalar.

The concept of the mass of an isolated gravitational system was defined by Arnowitt-Deser-Misner (ADM) in 1962 as an asymptotic value of the gravitational flux through spheres expanding out to infinity. As a special case, one may assume the spacelike manifold is time

Mu-Tao Wang is assistant professor of mathematics at Columbia University. His email address is mtwang@math. columbia.edu.

Shing-Tung Yau is professor of mathematics at Harvard University. His email address is yau@math. harvard. edu.

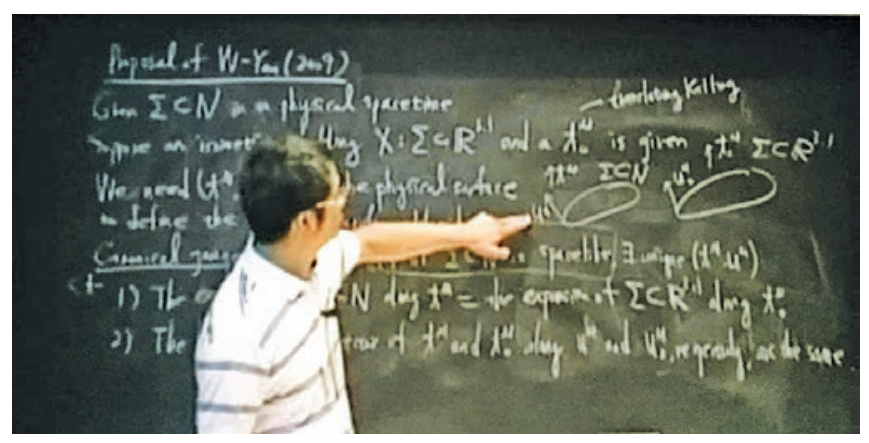

Mu-Tao Wang presenting at MSRI in 2014. The complete video of this introductory lecture on quasi-local mass is available at https: //www. msri.org/workshops/691.

symmetric and asymptotically flat and satisfies the dominant energy condition. So it is an asymptotically flat three-dimensional Riemannian manifold with nonnegative scalar curvature. The positive mass theorem of the second author and Schoen, proven in 1979, states that the ADM mass of such a manifold is nonnegative and if the ADM mass is zero, then the manifold is flat Euclidean 3-space. ${ }^{2}$

A notion of quasi-local mass still needs to be defined for bounded regions in a manifold. In fact, in the special year in geometry of 1979 at the Institute for Advanced Study, Penrose announced that the first major problem in classical general relativity was the concept of quasilocal mass. Ideally such a notion should take on positive values on a three-dimensional manifold with nonnegative scalar curvature unless the interior of the region is flat. It should asymptotically approach the ADM mass for spheres expanding to infinity. It should depend only upon the local properties of the boundary surface of the region, much as a flux depends only upon such properties.

One notion of quasi-local mass proposed by BrownYork in 1991-93 applies the work of Nirenberg-Pogorelov. The Brown-York quasi-local mass of a region is found by taking the difference of the total mean curvature of the surface's unique isometric embedding into Euclidean three-dimensional space and subtracting the total mean curvature of the surface as it lies in the spacelike manifold. Brown-York made this important step towards the definition of quasi-local mass by finding a surface Hamiltonian expression, which was derived from the Hilbert-Einstein functional. Their original version of the definition appeared to be gauge dependent (as it depended upon the three-manifold the surface enclosed). Many important works appeared immediately, including a key paper of Hawking-Horowitz in 1996.

Shi and Tam proved in 2002 that the Brown-York mass of a region in a three-dimensional manifold with nonnegative scalar curvature is nonnegative. Moreover, if the Brown-York mass is zero, then the region is isometric to the region lying within the Nirenberg-Pogorelov embedding into Euclidean space. It is interesting to note

\footnotetext{
${ }^{2}$ For simplicity we discuss only three dimensions here.
} 
that Shi-Tam were unaware of the work of Brown-York and the applications to general relativity at the time and were concerned only with manifolds of nonnegative scalar curvature and the work of Nirenberg-Pogorelov.

In general relativity it is essential to study a wide class of spacelike manifolds, not just those which are time symmetric and satisfy the positive energy condition (and thus have positive scalar curvature). In general such spacelike manifolds in spacetime have both a metric tensor and a second fundamental form. They may be viewed as initial data sets, and indeed Choquet-Bruhat proved in 1952 the existence and uniqueness of solutions to Einstein's equation given such initial data. A notion of quasi-local mass of a region in such a manifold should depend only upon this data on the boundary surface of the region. It should be zero when the manifold is a spacelike submanifold of flat Minkowski space (which has no matter field and no gravitation).

Liu-Yau introduced a quasi-local mass that was proven to be positive using Shi-Tam's proof and both SchoenYau's proof and Witten's proof of the positive mass theorem. However, a simple calculation shows that a surface in the light cone of the Minkowski spacetime has strictly positive Brown-York and Liu-Yau mass unless it is a round sphere.

Examining this problem together, it became clear to us that isometric embeddings into the Minkowski spacetime need to be taken into account in order to obtain a mass expression that satisfies the last criterion. By a simple degree of freedom counting, this yields an underdetermined problem, and an additional equation is needed to expect any type of uniqueness. On the other hand, the surface Hamiltonian expression relies on a definite choice of gauge choice, without which the definition remains ambiguous. We eventually came up with a variational approach to tackle this problem.

Nirenberg-Pogorelov's isometric embedding theorem allowed us to identify the extra degree of freedom as the time function. Returning to the derivation of Brown-York, we discovered that there is indeed a canonical choice of gauge with respect to each time function, to which a quasilocal energy (instead of mass) can be assigned. The choice of this gauge is justified by the positivity of the quasi-local energy, whose proof comprises ideas from Schoen-Yau, Shi-Tam, Bartnik, Witten, and Liu-Yau. We later realized the choice is closely related to a gravitational conservation law which plays an important role in the study of the dynamics of the Einstein equation.

With this positivity, we minimize the energy with respect to different time functions and define the quasilocal mass as the energy at critical points. This is very much like the rest mass of special relativity which realizes the minimal energy seen among all observers. The EulerLagrange equation is a fourth-order nonlinear equation of the time function. Together with the isometric embedding equation, this gives a well-determined system of four equations for four unknowns. This work was published in 2009.

We do not yet have a general existence or uniqueness theorem for this system. However, when the quasi-local mass is positive, the system is elliptic and the linearized equation can be solved. This is sufficient for studying many unsettled problems for isolated gravitating systems, on which most current study of general relativity focuses. We expect more applications to come when this optimal isometric embedding is better understood.

\section{On a Personal Note by $M$. T. Wang}

I was once invited to Professor Nirenberg's apartment more than ten years ago. I explained my work on higher codimensional mean curvature flows and minimal surface systems to him. He was quite surprised at first and was very attentive to the detail of my explanation. Overall, it was a very encouraging and inspiring experience for me.

\section{On a Personal Note by S. T.-Yau}

In 1974, Calabi and Nirenberg announced that they had solved the boundary value problem for the real Monge-Ampère equation. S. Y. Cheng and I also thought we had a solution. Chern told us that he was told that there was an error in the Calabi-Nirenberg solution, so he met with us and with Nirenberg to go over our proof. Still it turned out Shing-Tung Yau.



the boundary estimates were not strong enough to solve the problem. Three years later I told Nirenberg that Cheng and I could solve the problem without the strong boundary estimate. We proved the solution exists and is smooth in the interior of the domain while only $C^{1,1}$ up to the boundary. Nirenberg made a joke that we are like "half defeated generals." But for me, the solution is good enough to solve our geometric problem on affine spheres. After about eight years, Nirenberg finished the strong boundary estimate with Luis Caffarelli and Joel Spruck. I learned a lot from him in the process of those ten years, and he has always been encouraging.

\section{References}

[1] F. LABOURIE, Immersions isometriques elliptiques et courbes pseudo-holomorphes. (French) [Elliptic isometric immersions and pseudoholomorphic curves], J. Differential Geom. 30 (1989), no. 2, 395-424.

[2] C.-C. M. LIU and S.-T. YAU, Positivity of quasi-local mass II, J. Amer. Math. Soc. 19 (2006), no. 1, 181-204.

[3] LOUIS NiRENBERG, The Weyl and Minkowski problems in differential geometry in the large, Comm. Pure Appl. Math. 6 (1953), 337-394.

[4] Y. SHI and L.-F. TAM, Positive mass theorem and the boundary behaviors of compact manifolds with nonnegative scalar curvature, J. Differential Geom. 62 (2002), no. 1, 79-125.

[5] M.-T. WANG and S.-T. YAU, Quasi-local mass in general relativity, Phys. Rev. Lett. 102 (2009), no. 2, no. 021101.

[6] __ Isometric embeddings into the Minkowski space and new quasi-local mass, Comm. Math. Phys. 288 (2009), no. 3, 919-942. 